\title{
All-optical, Non-volatile, Chalcogenide Phase-change Meta-switch
}

\author{
Behrad Gholipour ${ }^{1}$, Jianfa Zhang ${ }^{1}$, Jonathan Maddock ${ }^{1}$, Kevin F. MacDonald ${ }^{1}$, Daniel W. Hewak ${ }^{1}$, \\ and Nikolay I. Zheludev ${ }^{1,2}$ \\ 1. Optoelectronics Research Centre \& Centre for Photonic Metamaterials, University of Southampton, SO17 1BJ, UK \\ 2. Centre for Disruptive Photonic Technologies, Nanyang Technological University, Singapore 637371, Singapore
}

We show experimentally that bistable, optically-induced phase switching in germanium antimony telluride (GST) - a member of the Te-based chalcogenide alloy family upon which all of today's re-writable optical disc and phase-change RAM technologies are based - provides a platform for the engineering of non-volatile metamaterial transmission/reflection modulators (Fig. 1) for near- to mid-infrared wavelengths with thicknesses down to $1 / 27$ of the operating wavelength. These hybrid materials provide a robust and versatile platform for a new generation of optical switching and memory devices.

Switching optical signals on the nanoscale is a significant challenge: conventional modulators exploiting the Pockels or Kerr effects are based on interference over distances much longer than the wavelength, necessitating devices with dimensions of several centimetres in the propagation direction. Modulating a signal by controlling the absorption coefficient or refractive index of a medium also requires substantial propagation lengths over which amplitude/phase changes accumulate, or interferometric arrangements that are inherently longer than the wavelength.

Here we demonstrate a robust and highly adaptable alternative based on the fact that the resonant optical properties of planar plasmonic metamaterials depend strongly on the near-field dielectric environment: small changes in the refractive index or absorption coefficient of an adjacent chalcogenide nano-layer (associated with optically-induced transitions between its amorphous and crystalline states) produce massive changes in the transmission and reflection characteristics of the hybrid metamaterial device ('meta-device') structure.

Experimental structures (Fig. 1a) comprised: a calcium fluoride or fused quartz substrate; a $50 \mathrm{~nm}$ thick gold metamaterial film (patterned by focused ion beam milling); a functional 15-40 nm film of GST and inert $\mathrm{ZnS} / \mathrm{SiO}_{2}$ buffer and capping layers either side of the chalcogenide. Phase transitions in both the forward (amorphous-crystalline) and reverse (crystalline-amorphous) directions were initiated uniformly across large $\left(\sim 2000 \mu \mathrm{m}^{2}\right)$ areas of the GST film by single-pulse laser excitation. Phase switching in the GST layer produces marked changes in its refractive index and shifts the resonance frequency of the metamaterial, bringing about a substantial change in optical properties of the meta-device at wavelengths close to the resonance (Fig. 1b).
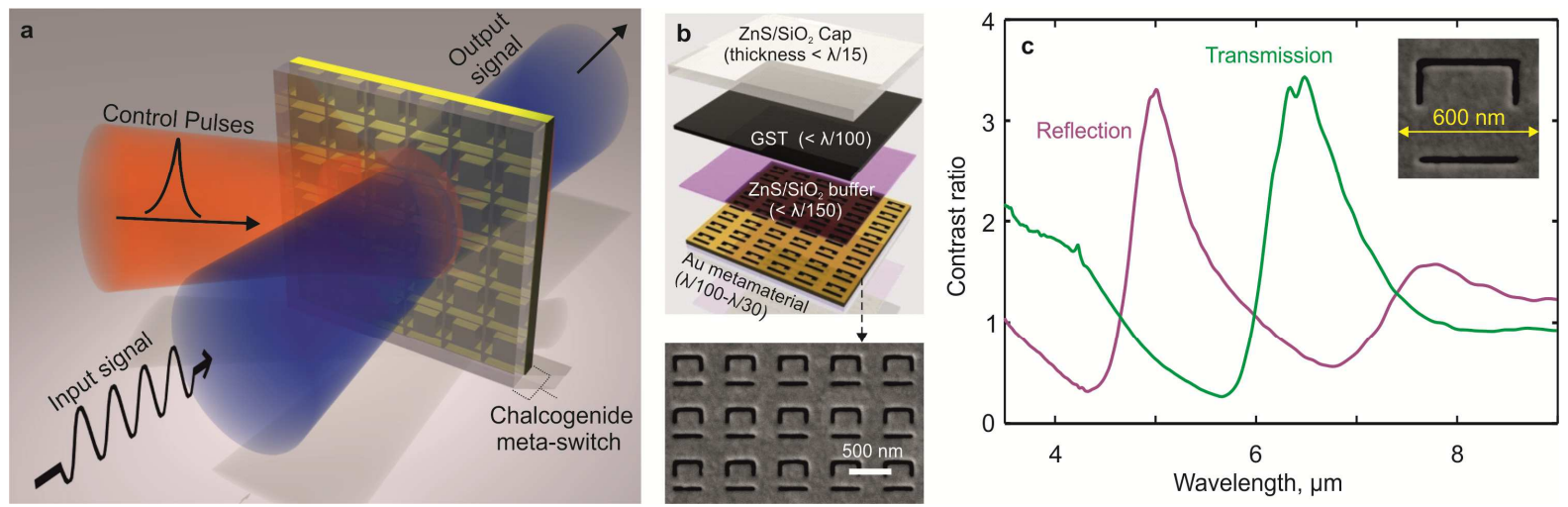

Fig. 1 (a) Artistic impression of an all-optical, non-volatile, chalcogenide meta-switch. (b) Multilayer structure of near- to mid-IR chalcogenide hybrid metamaterial switches (indicative layer thicknesses in brackets). (c) Spectral dispersion of transmission and reflection modulation contrast associated with GST phase-switching in a chalcogenide meta-switch engineered for mid-IR operation. Detail of the $600 \mathrm{~nm}$ metamaterial unit cell is shown in the scanning electron microscope image inset.

Chalcogenide phase-change metamaterials can be switched electronically and thermally as well as optically to produce high-contrast intensity and phase modulation, and may be engineered to function at any visible to mid-infrared wavelength within the chalcogenide's transparency range. New glass compositions and excitation regimes may provide access to ultrafast and multi-level switching modalities. As such these structures offer a versatile platform for the development of a new generation of nanoscale optical switching, memory and spatial light modulation devices. 